\title{
Incorporating Unstructured Text in Multi-Layer Perceptron (MLP) Network: Factors Affecting Partner Selection in Pair Programming
}

\author{
Soo See Chai \\ Faculty of Computer Science and Information Technology \\ University Malaysia Sarawak (UNIMAS) \\ 94300, Kota Samarahan, Sarawak, Malaysia \\ sschai@unimas.my \\ Hui Hui Wang \\ Faculty of Computer Science and Information Technology \\ University Malaysia Sarawak (UNIMAS) \\ 94300, Kota Samarahan, Sarawak, Malaysia \\ hhwang@unimas.my
}

\author{
Kok Luong Goh \\ School of Science and Technology \\ International University College of Advanced Technology \\ Sarawak (i-CATS University College) \\ 93350, Kuching, Sarawak, Malaysia \\ klgoh@ppks.edu.my \\ Bui Lin Wee \\ Faculty of Computer Science and Information Technology \\ University Malaysia Sarawak (UNIMAS) \\ 94300, Kota Samarahan, Sarawak, Malaysia \\ blwee@unimas.my
}

\begin{abstract}
The revealed analysis studies on pair programming so far indicate that pair programming has produced affirmative effects on some aspects of students' performance. In the academic field, the usual practice of pair programming would be pairing the students in line with the programming skills of the students by the respective lecturers. This means, compatibility of the students in terms of their programming skills is the main focus when the pairing was done by the lecturers. Yet, research on elements that the students are looking into when they are given the liberty to decide on their partner in pair programming is lacking. In this study, a multi-layer perceptron (MLP) is developed to predict the preference of opting pair programming over solo programming. The Bayesian Information Criterion was used to select the best features in the prediction. The potential of unstructured text entered by the participants as comments in the questionnaire is incorporated in the MLP model to verify its capabilities towards prediction accuracy, i.e., to verify whether their comments are connected to their preference for pair programming versus solo programming. It was found that, when the students are given the freedom to choose their partner in pair programming, in the context of Malaysia, the students would pay attention to the ethnic criterion. This also suggests that the unstructured texts in the form of comments submitted by the participants in the questionnaire did not contribute to their choices on whether to undertake solo or pair programming.
\end{abstract}

Keywords-neural network, multi-layer perceptron, MLP, pair programming, unstructured text

\section{INTRODUCTION}

Learning to program is the foremost requirement in the field of computer science education. However, many students lost their interest in programming during the introductory programming courses. Pair programming is a technique that has emerged from the industry and has resulted in higher performance and retention in the learning of computer programming languages in universities and industries. In addition, this method of collaborative learning has exposed the participants in the pair to various ideas and increased the motivation of the partners [1]. As the name of pair programming implies, this technique is a collaborative form

Grant: UNIMAS F08/SoTL10/1349/2016(10). of programming in which two individuals work side by side using one computer. One of the two members is a programmer known as a "driver" who controls the keyboard and mouse, while another participant is the "navigator" who actively participates in the design and development of the program [2]. During the pair programming session, the driver and observer alternating their role periodically [3].

Despite the success and efficiency of pair programming practice, its benefits vary based on the essence of the partners exchanges and their former relationships [4]. Not all pair programming interaction is successful [5]. Poor experiences during pair programming could eventually deter the students from working in pairs [6]. If a partnership does not work out effectively, the pair would result in low throughput and may end up failing to accomplish the assignments [7, 8]. In reality, the success of pair programming depends heavily on how the partners in the pair interact [9]. Constant communication among the partners is highly encouraged throughout the pair programming session to exchange their ideas [10].

In the field of academics, students are normally paired with partners assigned by the lecturers according to the level of programming skill [11]. In another word, students are matched based on the perceived programming compatibility by their lecturers. It is reported that, when comparing students who are paired randomly and working solo, the performance of students who are matched using demonstrated ability performed better [12]. Another study on compatibility students pairing [13] showed that, majority of the students preferred to be matched with a partner who is compatible with them in terms of a similar grade point average. Yet, a study on workable pairs [14] discovered that encouraging students to select their own choice of partner managed to produce a lot of compatible pairings. In addition, allowing freedom of choosing pairing partners could minimize the time needed to find a suitable pair by the instructor and could result in peer support groups that would help the members in the course. Even so, methodologies employed in pair programming focus mainly on pairing the partners by the course instructor according to the perceived demonstrated skills. In this study, we will predict what are the factors that contribute to partner selection when students 